Y. Yamada

Nagoya Math. J.

Vol. 70 (1978), 111-123

\title{
PERIODIC SOLUTIONS OF GERTAIN NONLINEAR PARABOLIC DIFFERENTIAL EQUATIONS IN DOMAINS WITH PERIODICALLY MOVING BOUNDARIES
}

\author{
YOSHIO YAMADA
}

\section{Introduction}

In this paper we consider the periodic problems for certain nonlinear parabolic differential equations in domains with periodically moving boundaries. The typical problem, which is going to be discussed in the present paper, is to solve the following:

$$
\begin{cases}\frac{\partial u}{\partial t}-\sum_{i=1}^{n} \frac{\partial}{\partial x_{i}}\left(\left|\frac{\partial u}{\partial x_{i}}\right|^{p-2} \frac{\partial u}{\partial x_{i}}\right)+\beta(u) \ni f & \text { on } Q, \\ u(x, t)=0 & \text { on } \Gamma, \\ u(x, 0)=u(x, T) & \text { on } Q(0)=Q(T),\end{cases}
$$

where $\beta$ is a (possibly multi-valued) maximal monotone operator in $R^{1}$ $\times R^{1} ; Q$ is a bounded domain in $R_{x}^{n} \times(0, T)$ with the periodically moving boundary $\Gamma$ and $Q(t)$ is the section of $Q$ at $t$.

To approach our problems, we establish, in $\S 1$, the existence and uniqueness theorem (Theorem 1.4) for the abstract nonlinear differential equation of the form

$$
d u(t) / d t+\partial \varphi^{t}(u(t)) \ni f(t), \quad 0 \leqslant t \leqslant T,
$$

in a real Hilbert space $H$. Here, $\partial \varphi^{t}$ is the subdifferential of a lower semicontinuous convex function $\varphi^{t}$ from $H$ into $(-\infty,+\infty$ ] with the $t$ dependent effective domain $D\left(\varphi^{t}\right)$.

In $\S 2$, by use of the results in $\S 1$, we show the existence and uniqueness of periodic solutions for the above nonlinear parabolic differential equation and the modified Stokes equation in domains with periodically

Received November 8, 1976. 
moving boundaries. We employ the method used in the previous paper [8] and treat the problems directly in non-cylindrical domains without any change of variables.

The author wishes to express his hearty thanks to Professors $\mathrm{H}$. Fujita and K. Masuda for their kind advices and encouragements.

\section{§1. Abstract theory}

Let $H$ be a real Hilbert space with the inner product $(\cdot, \cdot)$ and the norm $\|\cdot\|$. Let $\left\{\varphi^{t}\right\}(0 \leqslant t \leqslant T)$ be a family of proper lower semicontinuous (l.s.c.) convex functions from $H$ into $(-\infty,+\infty]$. For each $0 \leqslant t$ $\leqslant T$, we put

$$
\begin{aligned}
D\left(\varphi^{t}\right) & =\left\{u \in H ; \varphi^{t}(u)<+\infty\right\}, \\
\partial \varphi^{t}(u) & =\left\{w \in H ; \varphi^{t}(v)-\varphi^{t}(u) \geqslant(w, v-u) \quad \text { for all } v \in D\left(\varphi^{t}\right)\right\}, \\
D\left(\partial \varphi^{t}\right) & =\left\{u \in D\left(\varphi^{t}\right) ; \partial \varphi^{t}(u) \neq \phi\right\} .
\end{aligned}
$$

The set $D\left(\varphi^{t}\right)$ is called the effective domain of $\varphi^{t}$ and the set $\partial \varphi^{t}(u)$ is called the subdifferential of $\varphi^{t}$ at $u$. It is well known that $\partial \varphi^{t}$ is a maximal monotone operator in $H \times H$.

In this section we consider the periodic problem for the abstract nonlinear differential equation associated with the time-dependent subdifferential operators;

$$
\left\{\begin{array}{l}
d u(t) / d t+\partial \varphi^{t}(u(t)) \ni f(t), \quad 0 \leqslant t \leqslant T, \\
u(0)=u(T) .
\end{array}\right.
$$

We now define a strong solution of (P.P).

Definition 1.1. Let $u:[0, T] \rightarrow H$. Then $u$ is called a strong solution of (P.P) if (i) $u \in C([0, T] ; H)$; i.e. $u$ is strongly continuous on [0,T], (ii) $u(0)=u(T)$, (iii) $u$ is strongly absolutely continuous on any compact subset of $(0, T)$ and (iv) $u(t)$ is in $D\left(\partial \varphi^{t}\right)$ for a.e. $t \in[0, T]$ and it satisfies

$$
d u(t) / d t+\partial \varphi^{t}(u(t)) \ni f(t) \quad \text { for a.e. } t \in[0, T] .
$$

In what follows, we often make the following assumptions (A.1)(A.3) on $\left\{\varphi^{t}\right\}(0 \leqslant t \leqslant T)$.

(A.1) There exist constants $\delta_{0}>0$ and $0 \leqslant \alpha \leqslant 1$ such that if $r>0$ and $0 \leqslant t_{0} \leqslant T$, then, for each $x_{0} \in D\left(\varphi^{t_{0}}\right)$ such that $\left\|x_{0}\right\| \leqslant r$, there exists an 
$H$-valued function $x$ on $I\left(t_{0}\right) \equiv\left[\max \left\{0, t_{0}-\delta_{0}\right\}, \min \left\{t_{0}+\delta_{0}, T\right\}\right]$ satisfying (i) $\left\|x(t)-x_{0}\right\| \leqslant\left|g_{r}(t)-g_{r}\left(t_{0}\right)\right|\left(\varphi^{t_{0}}\left(x_{0}\right)+K_{r}\right)^{\alpha} \quad$ for $t \in I\left(t_{0}\right)$, (ii) $\varphi^{t}(x(t)) \leqslant \varphi^{t_{0}}\left(x_{0}\right)+\left|h_{r}(t)-h_{r}\left(t_{0}\right)\right|\left(\varphi^{t_{0}}\left(x_{0}\right)+K_{r}\right) \quad$ for $t \in I\left(t_{0}\right)$, where $K_{r}$ is a non-negative constant and $g_{r}$ and $h_{r}$ are absolutely continuous functions on $[0, T]$ such that $g_{r}^{\prime} \in L^{2}(0, T)$ in case $0 \leqslant \alpha \leqslant 1 / 2$ and $g_{r}^{\prime} \in L^{1 / 1-\alpha}(0, T)$ in case $1 / 2<\alpha \leqslant 1$.

$$
\liminf _{\|u\| \rightarrow \infty} \frac{\varphi^{t}(u)}{\|u\|}=\ell(t), \quad 0 \leqslant t \leqslant T,
$$

where $\ell$ is a measurable function on $[0, T]$ such that $\int_{0}^{T} \ell(t) d t=+\infty$.

$$
D\left(\varphi^{T}\right) \subset D\left(\varphi^{0}\right)
$$

In order to show the existence of a strong solution of (P.P), we use the results on the initial value problem

$$
\left\{\begin{array}{l}
d u(t) / d t+\partial \varphi^{t}(u(t)) \ni f(t), \quad 0 \leqslant t \leqslant T, \\
u(0)=a .
\end{array}\right.
$$

As to the problem (I.V.P.) we have:

Proposition 1.2 (cf. Yamada [8, Theorem I]). Let $f \in L^{2}(0, T ; H)$ and let $\left\{\varphi^{t}\right\}(0 \leqslant t \leqslant T)$ satisfy the assumption (A.1). Then, for each $a \in \mathrm{Cl}\left(D\left(\varphi^{0}\right)\right)(\mathrm{Cl}=$ closure $)$ there exists a unique strong solution $u$ of (I.V.P) with the following properties.

(i) For every $0<t \leqslant T, u(t)$ is in $D\left(\varphi^{t}\right)$ and $\varphi^{t}(u(t))$ is absolutely continuous on $(0, T]$. Moreover, $\varphi^{t}(u(t)) \in L^{1}(0, T)$ and $t \varphi^{t}(u(t)) \in L^{\infty}(0, T)$.

(ii) $t^{1 / 2} d u / d t \in L^{2}(0, T ; H)$.

In particular, if $a \in D\left(\varphi^{0}\right)$, then $u$ satisfies:

(i)' For every $0 \leqslant t \leqslant T, u(t)$ is in $D\left(\varphi^{t}\right)$ and $\varphi^{t}(u(t))$ is absolutely continuous on $[0, T]$.

(ii)' $\quad d u / d t \in L^{2}(0, T ; H)$.

Proof. We can prove this proposition with a slight modification of the proof of Theorem I in [8].

Remark 1.3. The problem (I.V.P) has been considered by many authors under the various assumptions on $\left\{\varphi^{t}\right\}$ (see e.g. Kenmochi [5]). However, in this paper we put the assumption (A.1) on $\left\{\varphi^{t}\right\}$ so that 
we can apply the results to nonlinear parabolic differential equations in non-cylindrical domains.

Now using Proposition 1.2 we can obtain the following results on the problem (P.P).

THEOREM 1.4. Let $f \in L^{2}(0, T ; H)$ and let $\left\{\varphi^{t}\right\}(0 \leqslant t \leqslant T)$ satisfy the assumptions (A.1)-(A.3). Then there exists a strong solution $u$ of $(P . P)$ with the following properties.

(i) For every $0 \leqslant t \leqslant T, u(t)$ is in $D\left(\varphi^{t}\right)$ and $\varphi^{t}(u(t))$ is absolutely continuous on $[0, T]$.

(ii) $d u / d t \in L^{2}(0, T ; H)$.

In particular, if $\partial \varphi^{t}$ is strictly monotone for each $0 \leqslant t \leqslant T$, then the strong solution $u$ of $(P . P)$ is uniquely determined.

Remark 1.5. Nagai [7] also treated the problem (P.P) in the form of the variational inequality and showed the existence of a periodic solution under the appropriate assumptions on $\left\{\varphi^{t}\right\}$.

Proof of Theorem 1.4. The uniqueness is evident by the strict monotonicity of $\partial \varphi^{t}$.

We shall sketch only the proof of the existence part; the method of the proof is similar to that of Nagai [7, Theorem 1]. For each $a \in \mathrm{Cl}\left(D\left(\varphi^{0}\right)\right)$ let $u_{a}$ be the strong solution of (I.V.P). We define a singlevalued mapping $S$ by $S a=u_{a}(T)$ for $a \in \mathrm{Cl}\left(D\left(\varphi^{0}\right)\right)$. Then by Proposition 1.2, the assumption (A.3) and the monotonicity of $\partial \varphi^{t}$ we see that $S$ is the nonexpansive mapping such that $S\left(\mathrm{Cl}\left(D\left(\varphi^{0}\right)\right)\right) \subset D\left(\varphi^{0}\right)$. Therefore, if we can show that $S$ has a fixed point in $\mathrm{Cl}\left(D\left(\varphi^{0}\right)\right)$, then using Proposition 1.2 we obtain the conclusions of Theorem 1.4 .

To prove that $S$ has a fixed point in $\mathrm{Cl}\left(D\left(\varphi^{0}\right)\right)$ we use a fixed point theorem due to Browder and Petryshyn (see Lemma 1.6 below), which discusses the Picard iterates $\left\{x_{n}\right\}$ for $S$;

$$
x_{n+1}=S x_{n}, \quad x_{0} \in \mathrm{Cl}\left(D\left(\varphi^{0}\right)\right) \quad \text { given } .
$$

For brevity we put $u_{n}=u_{x_{n}}$. Noting $u_{n}(T)=S x_{n}$ we have

$$
\left\|u_{n}(T)-u_{n}(0)\right\|=\left\|S x_{n}-x_{n}\right\|=\left\|S^{n+1} x_{0}-S^{n} x_{0}\right\| \leq\left\|S x_{0}-x_{0}\right\|
$$

for all $n$. Consequently, the existence of a fixed point of $S$ in $\operatorname{Cl}\left(D\left(\varphi^{0}\right)\right)$ follows from the next two lemmas. 
Lemma 1.6 (Browder and Petryshyn [3, Theorem 1]). Let $S$ be a nonexpansive selfmapping of a nonempty closed convex set $C$ of $H$. Then $S$ has a fixed point in $C$ if and only if for any $x_{0} \in C$ the sequence of Picard iterates $\left\{x_{n}\right\}$ starting at $x_{0}$ (i.e. $x_{n+1}=S x_{n}$ ) is bounded in H.

Lemma 1.7 (cf. Benilan and Brezis [1, Lemma 3.1] and Nagai [6, Lemma 1]). Under the assumptions (A.1)-(A.2) let $f \in L^{2}(0, T ; H)$ and let $\left\{u_{n}\right\}$ be a sequence of strong solutions of the equations

$$
d u_{n}(t) / d t+\partial \varphi^{t}\left(u_{n}(t)\right) \ni f(t), \quad 0 \leqslant t \leqslant T,
$$

satisfying $\left\|u_{n}(0)\right\|-\left\|u_{n}(T)\right\| \leqslant C$ for all $n$. Then the sequence $\left\{u_{n}\right\}$ is bounded in $C([0, T] ; H)$.

Since Lemma 1.7 can be proved in the same way as Lemma 3.1 in [1], we complete the proof of Theorem 1.4 .

\section{§ 2. Applications}

In this section we apply Theorem 1.4 to the periodic problems for certain nonlinear parabolic differential equations in domains with periodically moving boundaries. We solve these problems directly in noncylindrical domains without any change of variables. Our main interest lies in the methodological part rather than in the specific results. We shall give two examples to explain how Theorem 1.4 is applied to them.

2.1. Nonlinear parabolic differential equation with principal part in divergence form

To treat the periodic problem stated in the introduction we take an appropriate Hilbert space and define a family of $t$-dependent l.s.c. convex functions $\left\{\varphi^{t}\right\}$. Then we formulate the original problem in the form (P.P) and solve (P.P).

Now we shall state the problem more precisely. Let $T$ be a positive number and, for each $0 \leqslant t \leqslant T$, let $Q(t)$ be a bounded domain in $R_{x}^{n}$ with the boundary $\Gamma(t)$. When $t$ moves over $(0, T), Q(t)$ generates an $(x, t)$-domain $Q=\bigcup_{0<t<T}(Q(t) \times\{t\})$ and $\Gamma(t)$ generates an $(x, t)$-hypersurface $\Gamma=\bigcup_{0<t<T}(\Gamma(t) \times\{t\}) ; \Gamma$ is the lateral boundary of $Q$. Assuming $Q(0)=Q(T)$ we consider the problem 


$$
\begin{cases}\frac{\partial u}{\partial t}-\sum_{i=1}^{n} \frac{\partial}{\partial x_{i}}\left(\left|\frac{\partial u}{\partial x_{i}}\right|^{p-2} \frac{\partial u}{\partial x_{i}}\right)+\beta(u) \ni f & \text { on } Q, \\ u=0 & \text { on } \Gamma, \\ u(x, 0)=u(x, T) & \text { on } Q(0)=Q(T),\end{cases}
$$

where $p \geq 2$ and $\beta$ is a (possibly multi-valued) maximal monotone operator in $R^{1} \times R^{1}$ such that $D(\beta) \ni 0$.

We now make the assumptions on the $(x, t)$-domain $Q$.

(Q.1) For each $0 \leqslant t \leqslant T, \Gamma(t)$ consists of $\ell$ simple closed hypersurfaces $\Gamma_{\alpha}(t)(\alpha=1,2, \cdots, \ell)$ which are sufficiently smooth (say, of class $\left.C^{2}\right)$ and satisfy

$$
\operatorname{dist}\left(\Gamma_{\alpha}(t), \Gamma_{\beta}(t)\right) \geqslant \varepsilon_{0} \quad \text { for every } 0 \leqslant t \leqslant T \text { and } \alpha \neq \beta,
$$

where $\varepsilon_{0}$ is a positive constant and dist $(\cdot, \cdot)$ denotes the $n$-dimensional distance.

(Q.2) Let $Q(s, t)=\bigcup_{s<r<t}(Q(r) \times\{r\})$. Then the $(x, t)$-domain $Q$ is covered by $m$ slices $Q\left(t_{i}, t_{i}+\delta_{i}\right)\left(\delta_{i}>0, i=1,2, \cdots, m\right)$ such that, for each $1 \leq i \leq m, Q\left(t_{i}, t_{i}+\delta_{i}\right)$ is mapped onto a cylindrical domain $Q\left(t_{i}\right) \times\left(t_{i}\right.$, $t_{i}+\delta_{i}$ ) by a diffeomorphism $\Phi_{i}$ which is smooth (say, of class $C^{3}$ ) up to the boundary and preserves the time coordinate $t$.

Remark 2.1. The assumption (Q.2) is satisfied, if for each $1 \leq \alpha \leq \ell$, $\Gamma_{\alpha}=\bigcup_{0<t<T}\left(\Gamma_{\alpha}(t) \times\{t\}\right)$ is covered by a finite number of open patches and, in each patch, $\Gamma_{\alpha}$ can be represented by $x_{n}^{\prime}=\psi\left(x_{1}^{\prime}, x_{2}^{\prime}, \cdots, x_{n-1}^{\prime}, t\right)$ under a suitable choice of coordinates $\left(x_{1}^{\prime}, x_{2}^{\prime}, \cdots, x_{n}^{\prime}\right)$ in $R^{n}$, where $\psi$ is a smooth function (say, of class $C^{4}$ ) of $n$ variables $\left(x_{1}^{\prime}, x_{2}^{\prime}, \cdots, x_{n-1}^{\prime}, t\right.$ ) (cf. Fujita [4]).

To apply Theorem 1.4 to the problem (2.1) we take an open ball $B$ in $R_{x}^{n}$ such that the closure of $Q(t)$ (in $R_{x}^{n}$ ) is contained in $B$ for every $0 \leq t \leq T$ and treat the problem (2.1) in $L^{2}(B)$. In this section we use the following notations; when $u$ is a real-valued function on $Q$, we put

$$
\begin{aligned}
& \hat{u}=\text { the natural extension of } u \text {, i.e., } \hat{u}=u \text { on } Q \\
& \quad \text { and } \hat{u}=0 \text { on }\{B \times(0, T)\} \backslash Q,
\end{aligned}
$$

and define the spaces

$$
\begin{aligned}
C\left([0, T] ; W_{0}^{1, p}(Q(t))\right)= & \left\{u ; \hat{u} \in C\left([0, T] ; W_{0}^{1, p}(B)\right) \text { and } u(\cdot, t)\right. \\
& \left.\in W_{0}^{1, p}(Q(t)) \text { for every } 0 \leqslant t \leqslant T\right\},
\end{aligned}
$$


and so on.

Now we note that we may assume $\beta(0) \ni 0$ without loss of generality. Therefore, since $\beta$ is a maximal monotone operator in $R^{1} \times R^{1}$ such that $\beta(0) \ni 0$, we can find a proper l.s.c. convex function $\gamma$ on $R^{1}$ satisfying $\partial \gamma=\beta$ and $\min \left\{\gamma(x) ; x \in R^{1}\right\}=\gamma(0)=0$.

Then we have the following theorem.

THEOREM 2.2. Let $f \in L^{2}(Q)$. Then there exists a unique solution $u$ of the problem (2.1) satisfying $u \in C\left([0, T] ; W_{0}^{1, p}(Q(t))\right), \gamma(u) \in L^{\infty}(0, T$; $\left.L^{1}(Q(t))\right)$ and $\partial u / \partial t \in L^{2}(Q)$.

Proof. To formulate the problem (2.1) in the form of (P.P) associated with subdifferential operators, we first define l.s.c. convex functions on $L^{2}(B)$ by

$$
\varphi_{B}(u)= \begin{cases}\frac{1}{p} \int_{B} \sum_{i=1}^{n}\left|\frac{\partial u}{\partial x_{i}}\right|^{p} d x & \text { if } u \in W_{0}^{1, p}(B) \\ +\infty & \text { if } u \in L^{2}(B), u \notin W_{0}^{1, p}(B)\end{cases}
$$

and

$$
\gamma_{B}(u)= \begin{cases}\int_{B} \gamma(u(x)) d x & \text { if } u \in L^{2}(B), \gamma(u) \in L^{1}(B) \\ +\infty & \text { if } u \in L^{2}(B), \gamma(u) \oplus L^{1}(B) .\end{cases}
$$

Then we can show $\partial \varphi_{B}=A_{B}$, where $A_{B}$ is a maximal monotone operator in $L^{2}(B) \times L^{2}(B)$ defined by

$$
A_{B} u=\mathscr{A} u \equiv-\sum_{i=1}^{n} \frac{\partial}{\partial x_{i}}\left(\left|\frac{\partial u}{\partial x_{i}}\right|^{p-2} \frac{\partial u}{\partial x_{i}}\right)
$$

with the domain $D\left(A_{B}\right)=\left\{u \in W_{0}^{1, p}(B) ; \mathscr{A} u \in L^{2}(B)\right.$ in the sense of the distribution\} (cf. Lions [6, chap. 2,2]). We can also show $\partial \gamma_{B}=\beta_{B}$, where $\beta_{B}$ is a maximal monotone operator in $L^{2}(B) \times L^{2}(B)$ defined by

$$
\begin{aligned}
& D\left(\beta_{B}\right)=\left\{u \in L^{2}(B) ; \text { there exists a } v \in L^{2}(B)\right. \text { such that } \\
& \qquad(x) \in \beta(u(x)) \text { a.e. } x \in B\}, \\
& \beta_{B}(u)=\left\{v \in L^{2}(B) ; v(x) \in \beta(u(x)) \text { a.e. } x \in B\right\} \quad \text { for } u \in D\left(\beta_{B}\right) .
\end{aligned}
$$

Moreover, using the results of Brezis [2, Proposition 2.17] we see that $A_{B}+\beta_{B}$ is also a maximal monotone operator in $L^{2}(B) \times L^{2}(B)$ which satisfies 


$$
\left\|\beta_{B}^{0}(u)\right\|_{L^{2}(B)} \leqslant\left\|\left(A_{B}+\beta_{B}\right)^{0}(u)\right\|_{L^{2}(B)} \quad \text { for } u \in D\left(A_{B}\right) \cap D\left(\beta_{B}\right) .
$$

We next define a closed convex subset $K(t)$ of $L^{2}(B)$ by

$$
K(t)=\left\{u \in L^{2}(B) ; u(x)=0 \text { a.e. } x \in B \backslash Q(t)\right\}
$$

and denote its indicator function by $I_{K(t)}$; i.e., $I_{K(t)}(u)=0$ if $u \in K(t)$ and $I_{K(t)}(u)=+\infty$ if $u \notin K(t)$.

We put $\varphi^{t}(u)=\varphi_{B}(u)+\gamma_{B}(u)+I_{K(t)}(u)$. Then $\varphi^{t}$ is a proper l.s.c. convex function on $L^{2}(B)$ with the effective domain $D\left(\varphi^{t}\right)=W_{0}^{1, p}(B)$ $\cap D\left(\gamma_{B}\right) \cap K(t)$. Since we can show by the assumption (Q.1) that $u \in D\left(\varphi^{t}\right)$ if and only if $\left.u\right|_{B \backslash Q(t)}=0$ and $\left.u\right|_{Q(t)} \in W_{0}^{1, p}(Q(t)) \cap D\left(\gamma_{Q(t)}\right)$, we see that $\varphi^{t}(u)$ is equal to $\varphi_{Q(t)}\left(\left.u\right|_{Q(t)}\right)+\gamma_{Q(t)}\left(\left.u\right|_{Q(t)}\right)$ for each $u \in D\left(\varphi^{t}\right)$. Therefore, we find that, when $u$ and $f$ are in $L^{2}(B)$,

$$
\begin{gathered}
\left\{\begin{array}{l}
u \in D\left(\partial \varphi^{t}\right) \\
f \in \partial \varphi^{t}(u)
\end{array}\right. \text { if and only if } \\
\left\{\begin{array}{l}
\left.u\right|_{B \backslash Q(t)}=0 \\
\left.u\right|_{Q(t)} \in D\left(A_{Q(t)}\right) \cap D\left(\beta_{Q(t)}\right) \\
\left.\left.f\right|_{Q(t)} \in A_{Q(t)} u\right|_{Q(t)}+\beta_{Q(t)}\left(\left.u\right|_{Q(t)}\right) .
\end{array}\right.
\end{gathered}
$$

(Note that $A_{Q(t)}+\beta_{Q(t)}$ is a maximal monotone operator in $L^{2}(Q(t)$ ) $\times L^{2}(Q(t))$.) Consequently we can formulate the problem (2.1) in the form

$$
\left\{\begin{array}{l}
d \hat{u}(t) / d t+\partial \varphi^{t}(\hat{u}(t)) \ni \hat{f}(t), \quad 0 \leqslant t \leqslant T, \\
\hat{u}(0)=\hat{u}(T),
\end{array}\right.
$$

where $\hat{f}$ is the natural extension of $f$. The required solution $u$ of (2.1) is given by $u=\left.\hat{u}\right|_{Q}$.

Now we shall verify the assumptions of Theorem 1.4. We begin with the verification of the assumption (A.1). Let $t_{0} \in[0, T]$ be fixed. Then by the assumption (Q.2) there exists a positive constant $\delta_{0} \leqslant \min _{1 \leqslant i \leqslant m} \delta_{i}$ such that

$$
Q\left(\max \left\{t_{0}-\delta_{0}, 0\right\}, \min \left\{t_{0}+\delta_{0}, T\right\}\right) \subset Q\left(t_{i}, t_{i}+\delta_{i}\right) \quad \text { for some } i .
$$

Since $Q\left(t_{i}, t_{i}+\delta_{i}\right)$ is mapped onto the cylindrical domain $Q\left(t_{i}\right) \times\left(t_{i}, t_{i}+\delta_{i}\right)$ by the diffeomorphism $\Phi_{i}$ which preserves the time coordinate $t$, the image $(\xi, \tau) \in Q\left(t_{i}\right) \times\left(t_{i}, t_{i}+\delta_{i}\right)$ of $(x, t) \in Q\left(\max \left\{t_{0}-\delta_{0}, 0\right\}, \min \left\{t_{0}+\delta_{0}, T\right\}\right)$ 
by $\Phi_{i}$ is represented by

$$
\xi=X(x, t) \text { and } \tau=t,
$$

where both $X$ and its inverse $X^{-1}$ are smooth functions up to the boundaries. We now take an arbitrary $v_{0} \in D\left(\varphi^{t_{0}}\right)$ and put for each $t \in I\left(t_{0}\right) \equiv\left[\max \left\{t_{0}-\delta_{0}, 0\right\}, \min \left\{t_{0}+\delta_{0}, T\right\}\right]$

$$
v(x, t)= \begin{cases}v_{0}\left(X^{-1}\left(X(x, t), t_{0}\right)\right) & \text { if } x \in Q(t) \\ 0 & \text { if } x \in B \backslash Q(t) .\end{cases}
$$

Then we can show that $v(\cdot, t)$ is in $D\left(\varphi^{t}\right)$ and that is satisfies

$$
\left\|v(\cdot, t)-v_{0}\right\|_{L^{2}(B)} \leqslant C_{1}\left|t-t_{0}\right| \varphi^{t_{0}}\left(v_{0}\right)^{1 / p}, \quad t \in I\left(t_{0}\right),
$$

and

$$
\varphi^{t}(v(\cdot, t)) \leqslant \varphi^{t_{0}}\left(v_{0}\right)+C_{2}\left|t-t_{0}\right| \varphi^{t_{0}}\left(v_{0}\right), \quad t \in I\left(t_{0}\right),
$$

for some positive constants $C_{1}$ and $C_{2}$ independent of $v_{0}$ and $t_{0}$. Thus the assumption (A.1) is verified.

We next verify the assumption (A.2). Let $t \in[0, T]$ and take $u \in D\left(\varphi^{t}\right)$. Since $\varphi^{t}(u)=\varphi_{Q(t)}\left(\left.u\right|_{Q_{(t)}}\right)+\gamma_{Q(t)}\left(\left.u\right|_{Q(t)}\right) \geqslant \varphi_{Q(t)}\left(\left.u\right|_{Q_{(t)}}\right)$, we obtain, by using Poincaré's inequality,

$$
\varphi^{t}(u) \geqslant C_{3}\|u\|_{L^{2}(Q(t))}^{p}=C_{3}\|u\|_{L^{2}(B)}^{p},
$$

where $C_{3}$ is a positive constant which depends only on $n, p$ and $B$. Hence, since $\varphi^{t}(u)=+\infty$ for $u \notin D\left(\varphi^{t}\right)$, we see that (2.5) holds for every $0 \leqslant t \leqslant T$ and $u \in L^{2}(B)$. Thus the assumption (A.2) is verified.

It is easy to verify the assumption (A.3).

Finally we shall show the strict monotonicity of $\partial \varphi^{t}$. For each $0 \leqslant t \leqslant T$ let $f_{i} \in \partial \varphi^{t}\left(u_{i}\right)(i=1,2)$ and $u_{1} \neq u_{2}$. Then by (2.3) we obtain

$$
\begin{aligned}
\left(f_{1}-f_{2}, u_{1}-u_{2}\right)_{L^{2}(B)}= & \left(f_{1}-f_{2}, u_{1}-u_{2}\right)_{L^{2}(Q(t))} \\
\geqslant & \left(A_{Q(t)} u_{1}-A_{Q(t)} u_{2}, u_{1}-u_{2}\right)_{L^{2}(Q(t))} \\
= & \int_{Q(t)} \sum_{j=1}^{n}\left(\left|\frac{\partial u_{1}}{\partial x_{j}}\right|^{p-2} \frac{\partial u_{1}}{\partial x_{j}}-\left|\frac{\partial u_{2}}{\partial x_{j}}\right|^{p-2} \frac{\partial u_{2}}{\partial x_{j}}\right) \\
& \times\left(\frac{\partial u_{1}}{\partial x_{j}}-\frac{\partial u_{2}}{\partial x_{j}}\right) d x \geqslant 0 .
\end{aligned}
$$

If $\left(f_{1}-f_{2}, u_{1}-u_{2}\right)_{L^{2}(B)}=0$ were true, then $\frac{\partial}{\partial x_{j}}\left(u_{1}-u_{2}\right)=0$ on $Q(t)$ for every $j$. Since $\left.u_{i}\right|_{Q(t)} \in W_{0}^{1, p}(Q(t))(i=1,2)$, we see $u_{1}=u_{2}$. Thus we have 
shown the strict monotonicity of $\partial \varphi^{t}$.

Therefore, applying Theorem 1.4 to (2.4) we see that there exists a unique strong solution $\hat{u} \in C\left([0, T] ; L^{2}(B)\right)$ of (2.4) with the following properties; (a) $\varphi^{t}(\hat{u}(t))$ is absolutely continuous in $0 \leqslant t \leqslant T$ and (b) $d \hat{u} / d t \in L^{2}\left(0, T ; L^{2}(B)\right)$. To obtain the conclusions of Theorem 2.2 , we have only to show the mapping $t \mapsto \hat{u}(t)$ is continuous on $[0, T]$ in the strong topology of $W_{0}^{1, p}(B)$. To prove this, we note that $\hat{u}(t)$ is in $D\left(\beta_{B}\right)$ for a.e. $t \in[0, T]$ by (2.3). Therefore, using the estimate (2.2) we deduce

$$
\begin{aligned}
\left\|\beta_{B}^{0}(\hat{u}(t))\right\|_{L^{2}(B)} & =\left\|\beta_{Q^{(t)}}^{0}(u(t))\right\|_{L^{2}(Q(t))} \\
& \leqslant\left\|\left(A_{Q(t)}+\beta_{Q(t)}\right)^{0}(u(t))\right\|_{L^{2}(Q(t))} \\
& \leqslant\|f(t)-d u(t) / d t\|_{L^{2}(Q(t))} \quad \text { for a.e. } t \in[0, T], \\
& =\|\hat{f}(t)-d \hat{u}(t) / d t\|_{L^{2}(B)} \quad \text { s. }
\end{aligned}
$$

from which it follows that $\beta_{B}^{0}(\hat{u})$ is in $L^{2}\left(0, T ; L^{2}(B)\right)$. Consequently, using the results of Brezis [2, Lemme 3.3] we obtain the absolute continuity in $t$ of $\gamma_{B}(u(t))$, which implies, together with the property (a), the absolute continuity of $\varphi_{B}(\hat{u}(t))$. Hence $\left(p \varphi_{B}(\hat{u}(t))\right)^{1 / p}=\|\hat{u}(t)\|_{W_{0}^{1}, p_{(B)}}$ is continuous in $0 \leqslant t \leqslant T$. Moreover, since $\hat{u} \in C\left([0, T] ; L^{2}(B)\right)$, we see that the mapping $t \mapsto \hat{u}(t)$ is continuous in the weak topology of $W_{0}^{1, p}(B)$. Hence $\hat{u}$ is in $C\left([0, T] ; W_{0}^{1, p}(B)\right)$ since the space $W_{0}^{1, p}(B)$ is uniformly convex. Thus we complete the proof of Theorem 2.2.

\subsection{Modified Stokes equation}

Under the same assumptions as in 2.1 we treat the periodic problem for the modified Stokes equation.

Let $\boldsymbol{u}=\left(u^{1}, u^{2}, \cdots, u^{n}\right)$ be an $n$-dimensional vector function and put

$$
\begin{gathered}
|\nabla \boldsymbol{u}|^{2}=\sum_{i, j=1}^{n}\left|\frac{\partial u^{j}}{\partial x_{i}}\right|^{2}, \\
\mathscr{A} \boldsymbol{u}=-\sum_{i=1}^{n} \frac{\partial}{\partial x_{i}}\left(|\nabla \boldsymbol{u}|^{p-2} \frac{\partial \boldsymbol{u}}{\partial x_{i}}\right), \quad p \geqslant 2 .
\end{gathered}
$$

We consider the problem

$$
\begin{cases}\frac{\partial u}{\partial t}+\mathscr{A} u=f-\operatorname{grad} p^{*} & \text { on } Q \\ \operatorname{div} \boldsymbol{u}=0 & \text { on } Q\end{cases}
$$




$$
\begin{cases}\boldsymbol{u}=0 & \text { on } \Gamma, \\ \boldsymbol{u}(x, 0)=\boldsymbol{u}(x, T) & \text { on } Q(0)=Q(T) .\end{cases}
$$

To apply Theorem 1.4 to the problem (2.6) we introduce the following notations; let $\Omega$ be a bounded domain in $R_{x}^{n}$ and put

$$
\begin{aligned}
& \boldsymbol{C}_{\sigma}^{\infty}(\Omega)=\left\{\boldsymbol{u}=\left(u^{1}, u^{2}, \cdots, u^{n}\right) ; u^{i} \in C_{0}^{\infty}(\Omega), \operatorname{div} \boldsymbol{u}=0\right\}, \\
& \boldsymbol{H}_{\sigma}(\Omega)=\text { the completion of } \boldsymbol{C}_{\sigma}^{\infty}(\Omega) \text { under the }\left(L^{2}(\Omega)\right)^{n} \text {-norm, } \\
& \begin{aligned}
W_{\sigma}^{1, p}(\Omega)= & \text { the completion of } \boldsymbol{C}_{\sigma}^{\infty}(\Omega) \text { under the }\left(W_{0}^{1, p}(\Omega)\right)^{n} \text {-norm, or } \\
& \text { equivalently } \\
= & \left\{\boldsymbol{u}=\left(u^{1}, u^{2}, \cdots, u^{n}\right) ; u^{i} \in W_{0}^{1, p}(\Omega), \operatorname{div} \boldsymbol{u}=0\right\},
\end{aligned} \\
& P_{\Omega}=\text { the orthogonal projection from }\left(L^{2}(\Omega)\right)^{n} \text { onto } \boldsymbol{H}_{\sigma}(\Omega) .
\end{aligned}
$$

Then as to the problem (2.6) we have:

THEOREM 2.3. Let $f \in\left(L^{2}(Q)\right)^{n}$. Then there exists a unique solution $\boldsymbol{u}$ of (2.6) satisfying $\boldsymbol{u} \in C\left([0, T] ; W_{\sigma}^{1, p}(Q(t))\right)$ and $\frac{\partial \boldsymbol{u}}{\partial t} \in\left(L^{2}(Q)\right)^{n}$.

Proof. As in 2.1 we introduce an auxiliary open ball $B \subset R_{x}^{n}$ and treat (2.6) in $H_{\sigma}(B)$. We first define a proper l.s.c. convex function on $\boldsymbol{H}_{\boldsymbol{\sigma}}(B)$ by

$$
\varphi_{B}(\boldsymbol{u})= \begin{cases}\frac{1}{p} \int_{B}|\nabla \boldsymbol{u}|^{p} d x & \text { if } \boldsymbol{u} \in \boldsymbol{W}_{\sigma}^{1, p}(B) \\ +\infty & \text { if } \boldsymbol{u} \in \boldsymbol{H}_{\sigma}(B), \boldsymbol{u} \oplus \boldsymbol{W}_{\sigma}^{1, p}(B) .\end{cases}
$$

Since $\mathscr{A}$ is a maximal monotone operator from $W_{\sigma}^{1, p}(B)$ on $W_{\sigma}^{1, p}(B)^{*}$ (the dual space of $W_{o}^{1, p}(B)$ ), we can show $\partial \varphi_{B}=A_{B}$, where $A_{B}$ is the restriction of $\mathscr{A}$ on $\boldsymbol{H}_{\sigma}(B) \times \boldsymbol{H}_{\sigma}(B)$ (cf. Lions [6, chap. 2,5]). We next define a closed convex subset $K(t)$ of $\boldsymbol{H}_{\sigma}(B)$ by

$$
\boldsymbol{K}(t)=\left\{\boldsymbol{u} \in \boldsymbol{H}_{\sigma}(B) ; \boldsymbol{u}=0 \text { a.e. on } B \backslash Q(t)\right\}
$$

and denote its indicator function by $I_{K(t)}$.

If we put $\varphi^{t}(\boldsymbol{u})=\varphi_{B}(\boldsymbol{u})+I_{\boldsymbol{K}(t)}(\boldsymbol{u})$, then we see from the assumption (Q.1) that, for each $0 \leqslant t \leqslant T, \boldsymbol{u} \in D\left(\varphi^{t}\right)$ if and only if $\left.\boldsymbol{u}\right|_{B \backslash Q(t)}=0$ and $\left.\boldsymbol{u}\right|_{Q(t)} \in W_{\sigma}^{1, p}(Q(t))$. Moreover, we can show that, when $\boldsymbol{u}$ and $\boldsymbol{f}$ are in $\boldsymbol{H}_{\sigma}(B)$, 


$$
\left\{\begin{array} { l } 
{ \boldsymbol { u } \in D ( \partial \varphi ^ { t } ) } \\
{ \boldsymbol { f } \in \partial \varphi ^ { t } ( \boldsymbol { u } ) }
\end{array} \text { if and only if } \left\{\begin{array}{l}
\left.\boldsymbol{u}\right|_{B \backslash Q(t)}=0 \\
\left.\boldsymbol{u}\right|_{Q(t)} \in D\left(A_{Q(t)}\right) \\
\left.P_{Q(t)} f\right|_{Q(t)}=\left.A_{Q(t)} \boldsymbol{u}\right|_{Q(t)}
\end{array} .\right.\right.
$$

Therefore, noting (2.7) we can formulate the problem (2.6) in the form

$$
\left\{\begin{array}{l}
d \hat{\boldsymbol{u}}(t) / d t+\partial \varphi^{t}(\hat{\boldsymbol{u}}(t)) \ni P_{B} \hat{f}(t), \quad 0 \leqslant t \leqslant T, \\
\hat{\boldsymbol{u}}(0)=\hat{\boldsymbol{u}}(T),
\end{array}\right.
$$

where $\hat{f}$ is the natural extension of $f$. The required solution $\boldsymbol{u}$ of (2.6) is given by $\boldsymbol{u}=\left.\hat{\boldsymbol{u}}\right|_{Q}$.

Now we shall verify the assumption (A.1). Let $t_{0} \in[0, T]$ be fixed and take an arbitrary $\boldsymbol{v}_{0}=\left(v_{0}^{1}, v_{0}^{2}, \cdots, v_{0}^{n}\right) \in D\left(\varphi^{t_{0}}\right)$. Using the same notations as in the proof of Theorem 2.2 we put for each $t \in I\left(t_{0}\right)$,

$$
\begin{aligned}
Y(x, t) & =X^{-1}\left(X(x, t), t_{0}\right) \\
a_{i j}(x, t) & =\frac{\partial Y_{i}}{\partial x_{j}}(x, t), \quad 1 \leqslant i, j \leqslant n .
\end{aligned}
$$

We denote by $\tilde{a}_{i j}(x, t)$ the $(i, j)$-cofactor of the $n \times n$-matrix $\left(a_{i j}(x, t)\right)$ and define

$$
v^{i}(x, t)= \begin{cases}\sum_{j=1}^{n} \tilde{a}_{j i}(x, t) v_{0}^{j}(Y(x, t)) & \text { if } x \in Q(t) \\ 0 & \text { if } x \in B \backslash Q(t) .\end{cases}
$$

Then $v(x, t)=\left(v^{1}(x, t), \cdots, v^{n}(x, t)\right)$ is in $D\left(\varphi^{t}\right)$ for each $t \in I\left(t_{0}\right)$. In fact,

$$
\begin{aligned}
&(\operatorname{div} \boldsymbol{v})(x, t)=\sum_{i, j=1}^{n}\{\left\{\frac{\partial \tilde{a}_{j i}}{\partial x_{i}}(x, t) v_{0}^{j}(Y(x, t))\right. \\
&\left.\quad+\sum_{k=1}^{n} \tilde{a}_{j i}(x, t) a_{k i}(x, t) \frac{\partial v_{0}^{j}}{\partial x_{k}}(Y(x, t))\right\} \\
&=|\operatorname{Jac} Y(x, t)|\left(\operatorname{div} \boldsymbol{v}_{0}\right)(Y(x, t))=0 .
\end{aligned}
$$

Here we used the equalities

$$
\begin{gathered}
\sum_{i=1}^{n} \frac{\partial \tilde{a}_{j i}}{\partial x_{i}}(x, t)=0, \quad 1 \leqslant j \leqslant n, \\
\sum_{i=1}^{n} \tilde{a}_{j i}(x, t) a_{k i}(x, t)=\delta_{j k}|\operatorname{Jac} Y(x, t)|, \quad 1 \leqslant j, k \leqslant n .
\end{gathered}
$$

Therefore, since it is clear that $\left.v(\cdot, t)\right|_{Q(t)}$ is in $\left(W_{0}^{1, p}(Q(t))\right)^{n}$, we see that $\boldsymbol{v}(\cdot, t)$ is in $D\left(\varphi^{t}\right)$. Moreover, we can show that $v(\cdot, t)$ satisfies 


$$
\left\|\boldsymbol{v}(\cdot, t)-\boldsymbol{v}_{0}\right\|_{\boldsymbol{H}_{\boldsymbol{\sigma}}(B)} \leqslant C_{4}\left|t-t_{0}\right| \varphi^{t_{0}}\left(\boldsymbol{v}_{0}\right)^{1 / p}, \quad t \in I\left(t_{0}\right),
$$

and

$$
\varphi^{t}(\boldsymbol{v}(\cdot, t)) \leqslant \varphi^{t_{0}}\left(\boldsymbol{v}_{0}\right)+C_{5}\left|t-t_{0}\right| \varphi^{t_{0}}\left(\boldsymbol{v}_{0}\right), \quad t \in I\left(t_{0}\right),
$$

for some positive constants $C_{4}$ and $C_{5}$ independent of $t_{0}$ and $v_{0}$. Thus the assumption (A.1) is verified.

The rest of the proof of this theorem is quite similar to that of Theorem 2.2; so we omit the details.

\section{Addendum}

After this paper was submitted for publication, the author was informed of the results of $\mathrm{A}$. Inoue and M. Wakimoto, "On existence of solutions of the Navier-Stokes equation in a time-dependent domain, J. Fac. Sci. Univ. Tokyo 24 (1977), 303-319". They treated the initial boundary value problem for the Navier-Stokes equation in a noncylindrical domain, on which they put similar assumptions to ours. However, their idea is quite different from ours. They transformed the original problem into the problem in a cylindrical domain by a suitable change of variables and obtained the existence result for the initial value problem.

\section{REFERENCES}

[1] P. Benilan and H. Brezis, Solutions faibles d'équations d'évolution dans les espaces de Hilbert, Ann. Inst. Fourier, Grenoble 22 (1972), 311-329.

[2] H. Brezis, Operateurs maximaux monotones et semi-groupes de contractions dans les espaces de Hilbert, North-Holland (1973).

[3] F. E. Browder and W. V. Petryshyn, The solution by iteration of nonlinear functional equations in Banach spaces, Bull. Amer. Math. Soc. 72 (1966), 571-575.

[4] H. Fujita, The penalty method and some nonlinear initial value problems, Contributions to Nonlinear Functional Analysis, edited by E. H. Zarantonello, Academic Press (1971), 635-665.

[ 5 ] N. Kenmochi, Some nonlinear parabolic variational inequalities, Israel J. Math. 22 (1975), 304-331.

[6] J. L. Lions, Quelques méthodes de résolution des problèmes aux limites non linéaires, Dunod Gauthier-Villars (1969).

[ 7 ] T. Nagai, Periodic solutions for certain time-dependent parabolic variational inequalities, Hiroshima Math. J. 5 (1975), 537-549.

[ 8 ] Y. Yamada, On evolution equations generated by subdifferential operators, J. Fac. Sci. Univ. Tokyo 23 (1976), 491-515. 
